\title{
Age Estimation Based on CLM, Tree Mixture With Adaptive Neuro Fuzzy, Fuzzy Svm
}

\author{
Mohammad Saber Iraji \\ Department of Computer Engineering and Information Technology, Payame Noor University, I.R. of Iran \\ Iraji.ms@gmail.com \\ Mohammad Bagher Iraji \\ Department of Electrical Engineering ,Phd student \\ mbiraji@gmail.com \\ Alireza Iraji \\ Department of Engineering, Damavand Branch, Islamic Azad University ,Science and Research Branch, \\ Damavand ,Iran \\ alireza.iraji@gmail.com \\ Razieh Iraji \\ Department of Engineering, Aliabadkatoul Branch, Islamic Azad University , aliabadkatoul ,Iran \\ Msriraji.ms@gmail.com
}

\begin{abstract}
As you know, age diagnosis based on the image is one of the most attractive topics in computer .In this paper, we present a intelligent model to estimate the age of face image. We use shape and texture feature extraction from FG-NET landmark image data set using AAM(Active Appearance Model), CLM (Constrained Local Model), tree Mixture algorithms. Finally, the obtained features were given as the training data to the ANFIS (adaptive neuro fuzzy influence system), FSVM(Fuzzy Support Vector Machine). Our experimental results show that In our proposed system, fuzzy svm has less errors and system worked more accurate and appropriative than prior methods. Our system is able to identify age of face image from different directions as is.
\end{abstract}

Index Terms - Face Age ,AAM, CLM, Tree Mixture ,Fuzzy Svm ,Anfis

\section{INTRODUCTION}

One of the main features of the human is age. In our society, we meet people with different age and sex, And we consider this factor in their behavior with others and We have good treatment of different age classes, For example, an old man to have a polite manner. Age also plays a role as a central feature of gender and race, are influenced by factors such as region climate, Health.

Find face and age estimation is one of the issues in machine vision. Facial image is very important subject because help in improving the human-machine action and reaction. Age prediction has many applications in systems security, parental control, finding missing persons search in large databases. A person's age can be guessed by the face image.
Sangeeta Agrawal, Rohit Raja, Sonu Agrawal[1] The method presented that applies Gaussian filtering for feature extraction with a Renyi entropy based SVM classification algorithm that adaptively Would improve the weights in the SVM decision function. Ages 0 to 69 years were considered. Age characteristics of AAM model of the shape model with 68 points on FG-net dataset.

Feng Gao, Haizhou Ai[10] Were used Gabor feature and fuzzy LDA through defining fuzzy age membership functions. They concluded that Gabor feature outperforms other features like pixel intensity and LBP the Fuzzy LDA can improve the classification Compared to SVM, AdaBoost and LDA and prediction performance get improved to $92.54 \%$ in their method.

Hironobu Fukai, Hironori Takimotoy, Yasue Mitsukura_ and Minoru Fukumi [9] Were designed age and gender estimation system by multiple features :shape feature, frequency feature, texture feature, color feature and estimate a age and gender using a neural network(NN).

Hamid Moghadam fard et all [8] propose a age-group estimation fuzzy method, extracted the image features using histograms of oriented gradients (HOG) and local binary patterns (LBP)and classified using neural networks and adaptive neuro-fuzzy inference system (ANFIS) the age-groups. Their results showed that the age group classification is performed with more accuracy.

Sarah N. Kohail [7] Applied Multi-layer perceptron neural networks (MLP) to estimate human ages from facial shape features extracted 94 points landmark image and were used Singular Value Decomposition (SVD) method.

Khoa luu ,karl ricanek jr nominate a age estimation technique that combine active appearance models(AAms) 
and support vector machines(SVM), used characteristics of face images as feature vectors $30 * 1$ by AAms, faces classify to adult and child. They to assign faces age use age function and apply mean absolute error(MAE) indicator[4].

Kwon, lobo used geometric ratio of key face feature and wrinkle for 47 image face, resulted in $100 \%$ accuracy [3].

Recent methods in Age estimation are local approaches and holistic approaches. One local approach methods is gaussian mixture model (GMMS),age features are such as :wrinkles on the forehead or at the eye corners[5].holistic approach use active appearance model(AAM) that AAM parameters extract from 68 point of landmark image and build a aging function: global aging function(GAF), Appearance Specific Aging Function (ASA), Weighted Appearance Aging Function (WAA), Weighted Person Specific Aging Function (WSA)[6].

David cristinacce and tim cootes present an efficient model (constrained local models) witch uses a joint shape and texture appearance model to create region template detectors. the model is fitted to unseen image, correlating the templates with target image to generate response and optimizing parameters the model is similar to that used in the AAM(active appearance model) but used feature templates instead of trying to approximate the image pixels directly. they say witch CLM algorithm is more accurate than original AAM method[2].

Xiangxin zhu, deva ramanan present a unified model for face detection, pose and landmark estimation based on a mixtures of trees with a shared pool on "in the wild "annotated dataset their tree model s was surprisingly effective in capturing elastic metamorphose[11].

Then, age estimation methods based on images of face have been widely perused.

The paper is organized in five sections. After the introduction in Section I, Section II introduces the existing methods of the pose estimation and landmark images with CLM. Section II continues with explanations of tree mixture in the wild algorithm in section III. in Section IV is proposed adaptive fuzzy neural network(anfis) and fuzzy svm age estimation model using CLM and tree mixture calibrated feature vectors. It continues with discussions on the architecture of hybrid learning and fuzzy model validation, the error of observations for training data sets. Section V presents the experimental results and conclusions of the research. The paper ends with a list of references.

\section{CLM}

There are many methods of computer vision that incorporate both shape and texture to build models and match to unseen images. CLM various uses template (rectangular regions) around a set of features, instead of triangular routes from the AAM with different search algorithms.

Other region templates approaches are Shape Optimized Search (SOS), Template Selection Tracker(TST), SMAT algorithm, Pictorial Structure
Matching (PSM) .CLM is an iterative scheme which obtain fit templates given the current feature points and target image. CLM is used statistical model to produce templates, while the AAM uses the invariant templates. we use the Viola and Jones algorithm to find face in image and combine PSM to incorporate initial feature points.

In CLM [2] The set of grey scale training vectors and normalized shape co-ordinates around each feature are applied to create linear models, from pixel values have zero mean and unit variance, As follows:

$\mathrm{x}={ }^{-} \mathrm{X}+\mathrm{P} s \mathrm{~b} s \quad \mathrm{~g}={ }^{-} \mathrm{g}+\mathrm{Pgb}$

Where $\mathrm{X}$ is a shape, $\mathrm{g}$ is intensity pattern vector of a training example, ${ }^{-} \mathrm{X}$ is the mean shape, Ps is a set of eigenvectors and bs is a set of shape parameters or vector of weights. Similarly ${ }^{-} \mathrm{g}$ is the mean normalized grey-level vector, $\mathrm{Pg}$ is a set of eigenvectors and bg is a set of grey-level parameters or vector of weights. The shape and template texture models are combined using a PCA to produce one joint model.

After the templates are applied to the image and compute response ,the position on feature points Are optimized Using objective function based on transfer function depended to bs shape parameters(Figure1). therefore CLM search algorithm As follows:

1. Input an initial feature points.

2. Repeat:

(a) Fit the joint model to the current feature points to construct a set of templates

(b) Use the shape optimizing search method to auspicate a new set of feature points.

Until Converged.

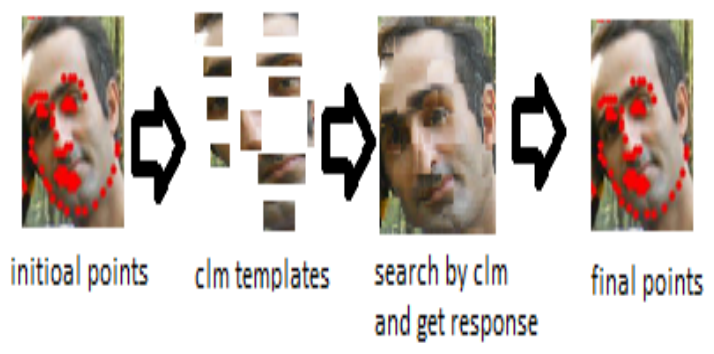

Figure 1. CLM Model

\section{TREE MIXTURE IN THE WILD}

This model is based on mixture of tree with a shared pool of parts $\mathrm{V}$ and sketch every facial landmark as part to obtain topological changes with global mixture. in the wild model can be globally optimized with dynamic programming and capture global elastic structure[11].Tree structure part model Can be formulated as follows:

$T_{m}=\left(V_{m}, E_{m}\right)$

where $m$ indicates a mixture and $V_{m}$ 
$\operatorname{shape}_{m}(L)=\sum_{i, j \in E_{m}} a_{i j}^{m} d x^{2}+b_{i j}^{m} d x+c_{i j}^{m} d y^{2}+$

$d_{i j}^{m} d y$

where $L=\left\{l_{i}: i \in V\right\}, d x=x_{i}-x_{j}, d=y_{i}-y_{j}$ and parameters $(a, b, c, d)$ determind the rest location of each spring.

Shape model can be rewritten as:

shape $_{m}(L)=-\left(L-M_{m}\right)^{T} \Lambda_{m}\left(L-M_{m}\right)+$ constant

where $L=\left\{l_{i}: i \in V\right\} ;(\Lambda, M) r e$

$$
\text { - parametrizations }
$$

of the shape model $(a, b, c, d)$

by labeled example $\left\{I_{n}\right\}$,we can write score as :

$\mathrm{S}(\mathrm{I}, \mathrm{z})=\beta \cdot \varphi(\mathrm{I}, \mathrm{z})$

where $z_{n}\left\{l_{n}, m_{n}\right\}, \varphi$ is feature from pixel location, $\beta$ is a parameters vector superseded $(a, b, c, d)$.

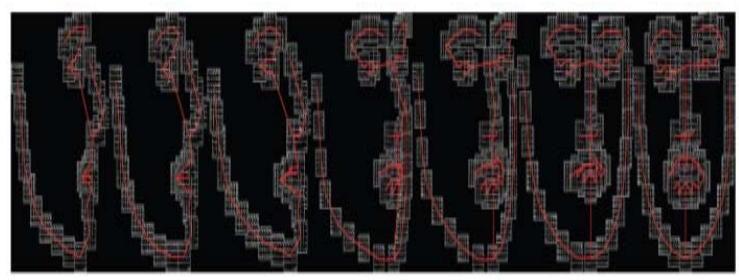

Figure 2. Mixture-of-Trees Model Encodes Topological

\section{THE PROPOSED MODEL FOR AGE ESTIMATION:}

As we mentioned, final training image can be represented by :

$X=X_{m}+P b$

, where $b$ is weights vector

The following equation was developed to approximate the age[6]:

$a g e=o f f s e t+W_{1} b^{T}+W_{2}\left(b^{2}\right)^{T}$

Where age is age of person in a face image.b , $\mathrm{b}^{2}$ are vectors containing 15 raw model parameters. $\mathrm{W}_{1}$, $\mathrm{W}_{2}$ are vectors containing weights for elements of $b, b^{2}$. Therefore, the age $=f(b)$.

After learning from training landmarked image, shape and texture weight parameters related to different methods (AAM, CLM, Mixture in the wild )are related to their optimal values. Consequently, we can be used the optimal parameter vector in each image as a feature vector to predict age. The feature vectors are considered as inputs to our neural network(Figure 3).

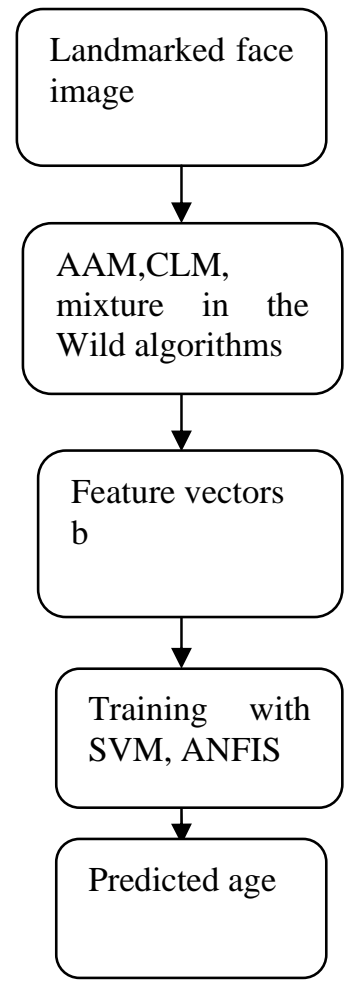

Figure 3. Proposed Model

Neural networks methods are based learning from historical data and fuzzy logic is a method that used to make logical decisions in an environment of doubt.

Activation function in neural networks can be a fuzzy membership function. The first efforts by ... Was performed (Lee \& Lee, 1975; see also Keller \& Hunt, 1985).

We used anfis and fuzzy svm neural networks for training and prediction in this research. The Support Vector Machines (SVMs), expand by Vapnik and coworkers, have been very successful in pattern classification. fuzzy set theory for SVM offered by Lin et al. and Huang et al.

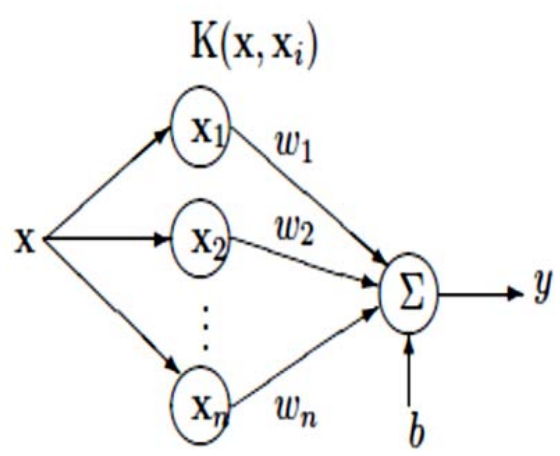

Figure 4. The Fuzzy Support Vector Machine Network

In Figure $4 \mathrm{~K}$ is the kernel of the transformation, xi is a support vector, $\mathrm{x}$ is an input vector, wi a weight, $\mathrm{b}$ the bias and $y$ the output of the network[1]. Knowledge of in each neuron are present the if -then rules. Each 
support vector displays a fuzzy rule. put $\mathrm{x}=[\mathrm{x} 1, \mathrm{x} 2, \ldots$ $\mathrm{xm}]$ an input vector, $\mathrm{xi}=[\mathrm{xi} 1, \mathrm{xi} 2, \ldots, \mathrm{xim}]$ a support vector and pi its response. A fuzzy rule can be introduced as:

IF $\mathrm{x} 1$ is circa $\mathrm{xi} 1$ AND $\mathrm{x} 2$ is circa $\mathrm{xi} 2$ AND $\mathrm{xm}$ is circa xim THEN $x$ belongs to pi.

But ANFIS (Adaptive Neuro Fuzzy Inference System) is based sugeno (Jang, Sun \& Mizutani, 1997; Jang \& Sun,1995). A typical rule in a Sugeno fuzzy model has the form If Input $1=\mathrm{x}$ and Input $2=\mathrm{y}$, then Output is $\mathrm{z}=\mathrm{ax}+\mathrm{by}+\mathrm{c}$. The following figure illustrates the anfis neural network[13]. In Figure 6 first layer are the degree of membership of linguistic variables, The second layer is 'rules layer' after the linear combination of rules at third layer then determine the degree of paraphernalia to a specific category by Sigmund function in layer 4 .

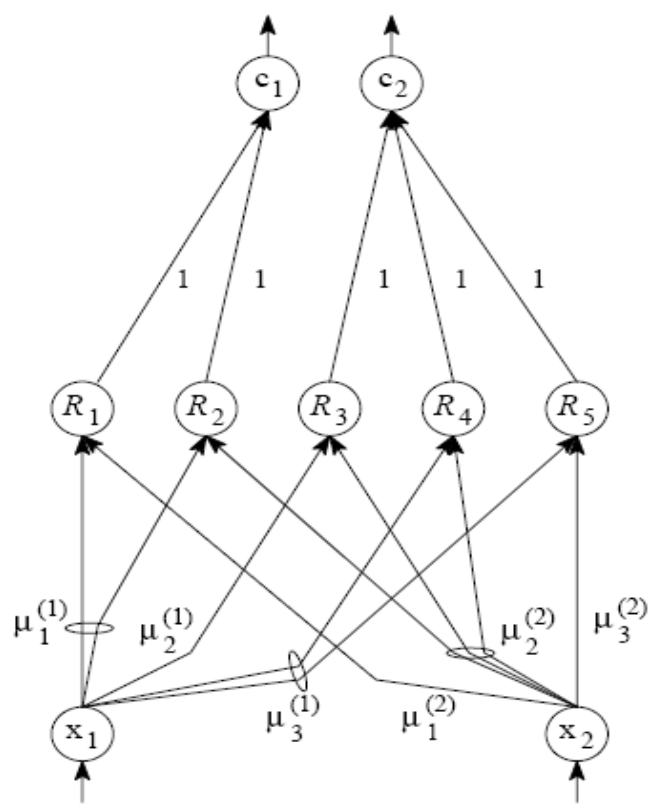

Figure 6 .Adaptive Neuro fuzzy Network(anfis)

\section{DISCUSSION AND CONCLUSION}

In this paper we recommend an age estimate fuzzy neural network The input features achieved with landmarked face image based on CLM ,mixture in the wild using fuzzy svm and anfis algorithms. We implement our proposed system in matlab and were used FG-NET data set contains 1002 landmark face images. A total of 400 images were used to train the proposed system and the remaining 602 images were used for testing.

Too our proposed system has the ability to predict the age of the images in different directions. The Figure 7 shows the output of the proposed algorithm on an image. The ages of 29 and 28 years were estimated. Some experimental results show in Figure 8,9,10.

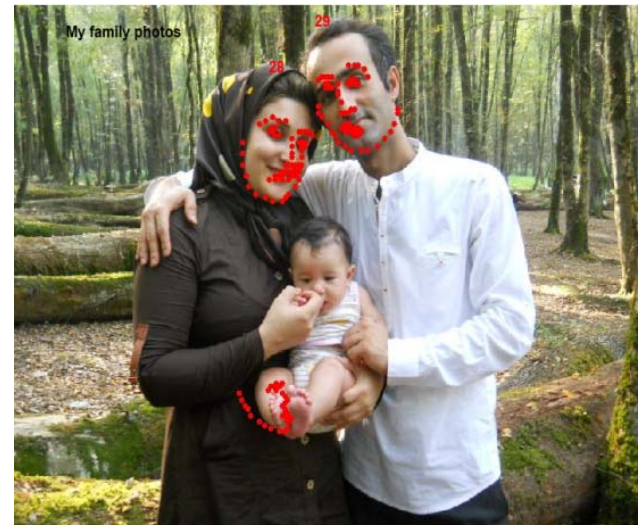

Figure 7. Output Of The Proposed Algorithm on an image

In order to compare of our proposed methods apply the rates Mean Absolate Error(MAE).

$M A E=\frac{\sum_{i=1}^{n} \mid \text { predicatedage }_{i}-\text { actualyage }_{i} \mid}{n}$

After the test MAE was calculated following Table I.

TABLE I. EXPRIMENTAL RESULTS MSE

\begin{tabular}{|c|c|c|}
\hline & anfis & Fuzzy svm \\
\hline AAM & 5.6 & 4.8 \\
\hline CLM & 4.58 & 4.25 \\
\hline Mixture in the wild & 3.96 & 3.72 \\
\hline
\end{tabular}

Our experimental results show that the fuzzy svm based on mixture in the wild approach is less error (3.72) and well performed other methods in determining the age of the show.

To future works can be used other neural networks like MLP to predict. It also color features and frequency features can to be used in combination with the texture and shape features from CML. We hope our system to be carefully by training from more face images.

\section{ACKNOWLEDGMENT}

This work received support from the Department of Computer Engineering and Information Technology, Payame Noor University ,I.R. of Iran. 


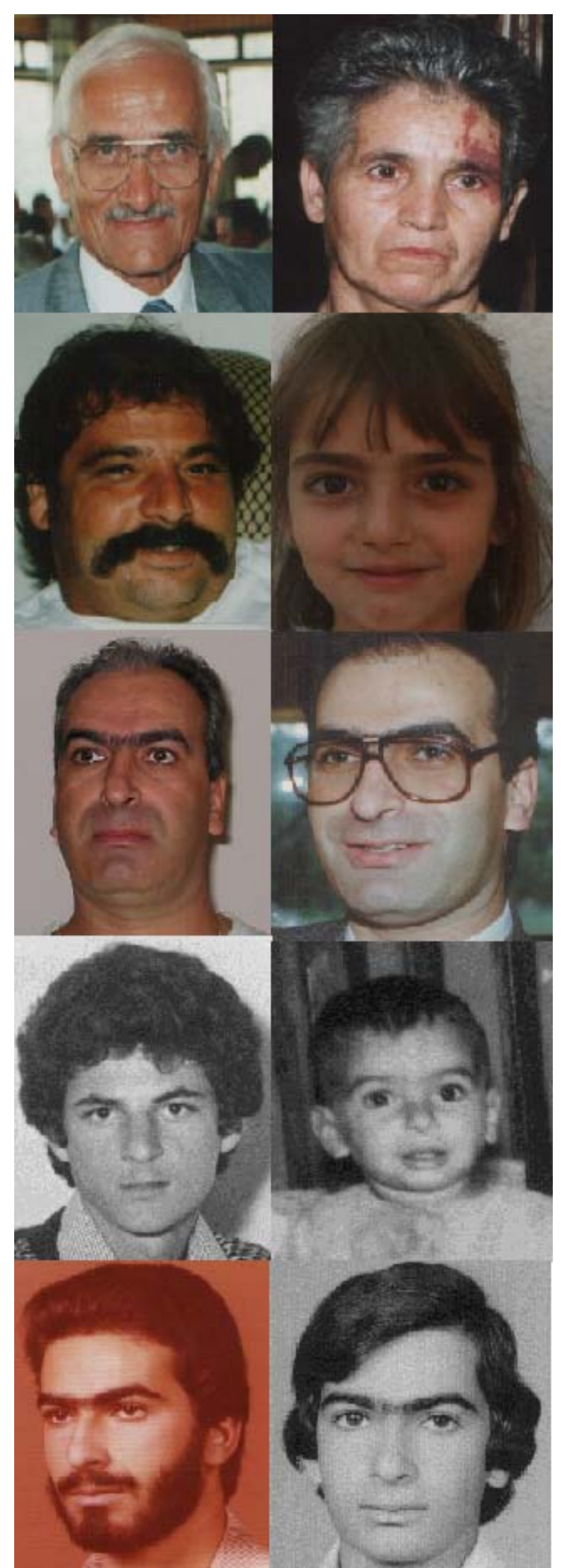

Figure 8. Some of FG-NET Data Set Original Images

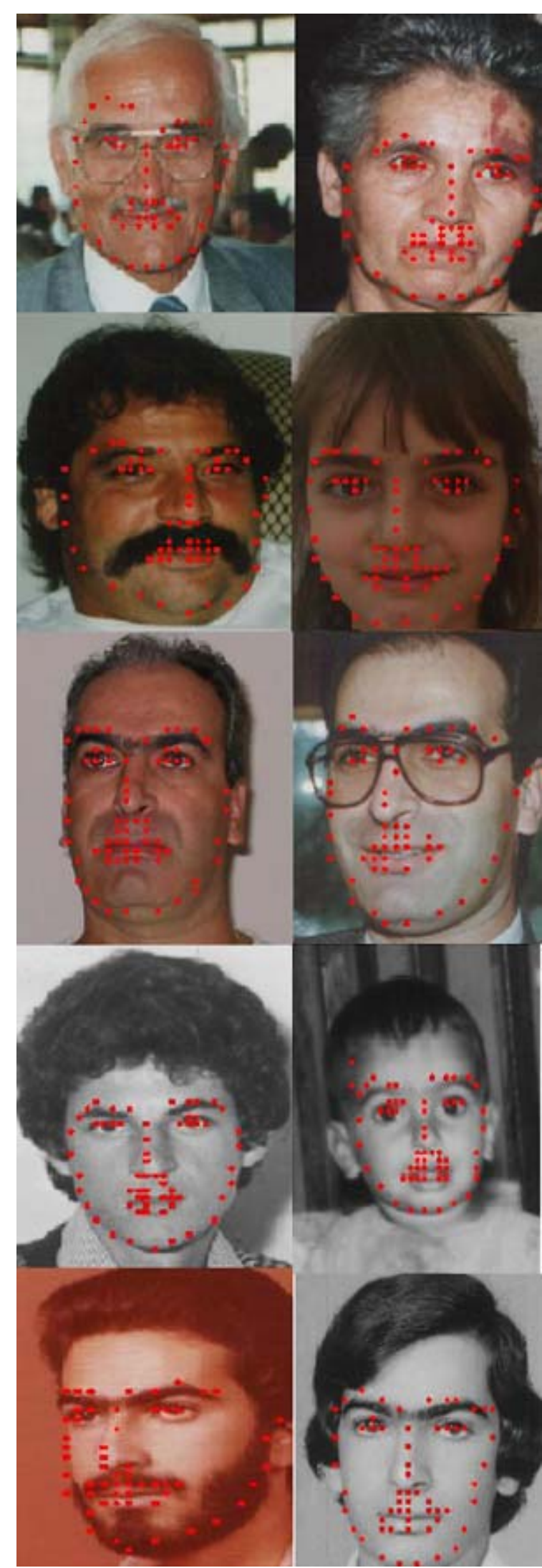

Figure 9. Output of Tree Mixture Algorithms on Example Showed in Figure 8 For Features Extraction(b vectores) and Input to Propsed Fuzzy Neural networks(FSVM,ANFIS) 


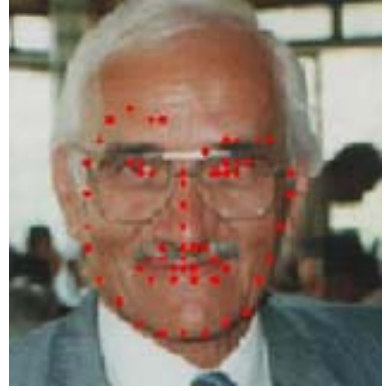

Actual age $=63$ years Predicted age $=61$ years

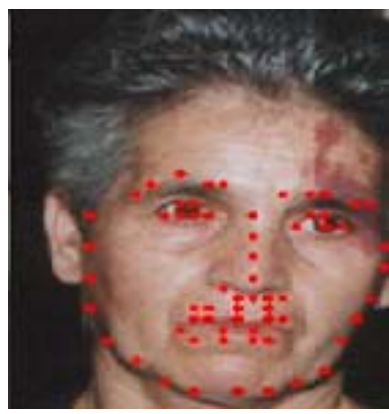

Actual age $=60$ years Predicted age $=62$ years

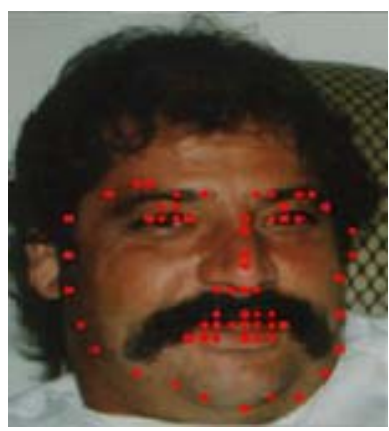

Actual age $=35$ years Predicted age $=36$ years

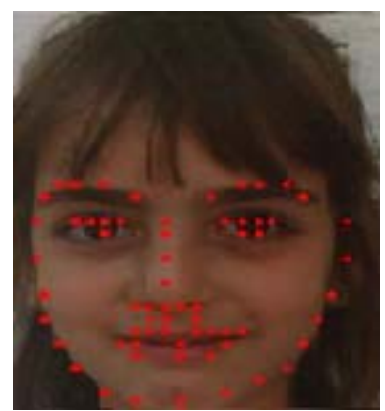

Actual age $=5$ years Predicted age $=5$ years

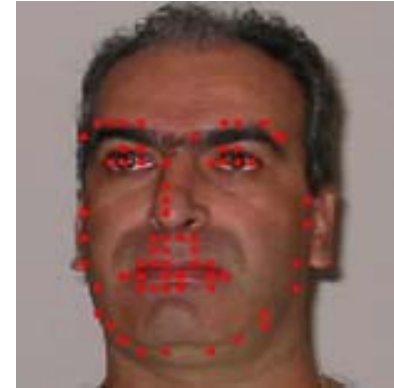

Actual age $=43$ years Predicted age $=42$ years

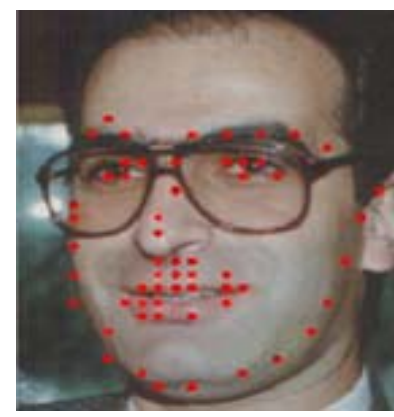

Actual age $=33$ years Predicted age $=34$ years

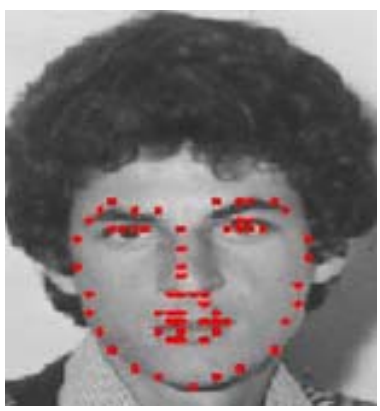

Actual age $=22$ years Predicted age $=22$ years

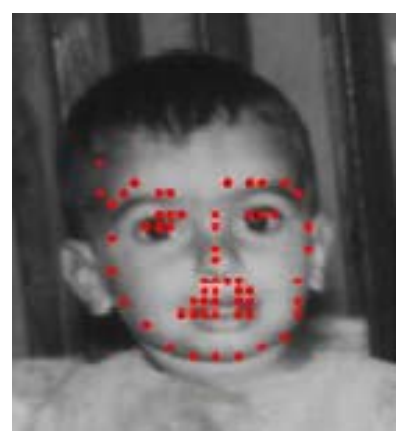

Actual age $=2$ years Predicted age $=1$ years 


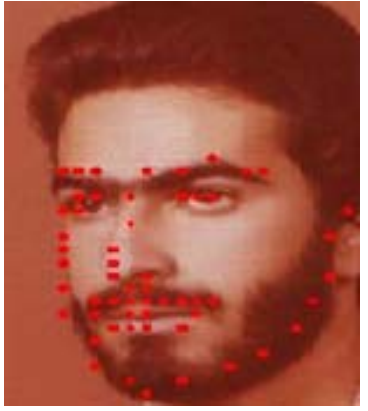

Actual age $=19$ years Predicted age $=21$ years

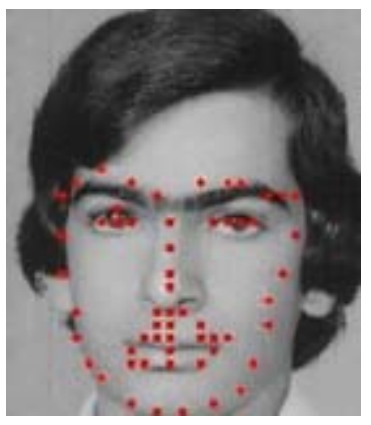

Actual age $=16$ years Predicted age $=16$ years

Figure 10. Output of Tree Mixture Algorithms on Example Showed in Figure 8 with FSVM and Compare Actual, Predicted age .

\section{REFERENCES}

[1] Sangeeta Agrawal, Rohit Raja, Sonu Agrawal," Support Vector Machine for age classification ”, International Journal of Emerging Technology and Advanced Engineering Website: www.ijetae.com (ISSN 2250-2459, Volume 2, Issue 5, May 2012).

[2] D Cristinacce, TF Cootes ,"Feature Detection and Tracking with Constrained Local Modes", Proc. British Machine Vision Conference, Vol. 3, pp.929-938, 2006.

[3] Y.h.kwon and n.da Vitoria lobo, "locating facial features for age classification”, Proc. SPIE 2055, Intelligent Robots and Computer Vision XII: Algorithms and Techniques, 62 (August 20, 1993).

[4] Khoa luu ,karl ricanek jr,” Age Estimation using Active Appearance Models and Support Vector Machine Regression”,IEEE 3rd International Conference on Biometrics: Theory, Applications, and Systems, 2009.

[5] S. Yan, M. Liu, T. S. Huang, Extracting Age Information from Local Spatially Flexible Patches, ICASSP, 2008.

[6] A. Lanitis, C. J. Taylor, T. F. Cootes, Modeling the process of ageing in face images, ICCV, 1999.

[7] Sarah N. Kohail,” Using Artificial Neural Network for Human Age Estimation Based on
Facial Images”, International Conference on Innovations in Information Technology (IIT), 2012.

[8] Hamid Moghadam fard, Sohrab Khanmohammadi, Sahraneh Ghaemi and Farshad Samadi," HUMAN AGE-GROUP ESTIMATION BASED ON ANFIS USING THE HOG AND LBP FEATURES”, Electrical and Electronics Engineering: An International Journal (ELELIJ) Vol 2, No 1, February 2013.

[9] Hironobu Fukai_, Hironori Takimotoy, Yasue Mitsukura_ and Minoru Fukumi," Age and Gender Estimation by using Facial Image ", The 11th IEEE International Workshop on Advanced Motion Control March 21-24, 2010, Nagaoka, Japan.

[10] Feng Gao, Haizhou Ai,” Face Age Classification on Consumer Images with Gabor Feature and Fuzzy LDA Method”, ICB '09 Proceedings of the Third International Conference on Advances in biometrics, 2009.

[11] Xiangxin zhu,deva ramanan," face detection, pose estimation, and landmark localization in the wild”, Computer Vision and Pattern Recognition (CVPR), IEEE Conference on Biometrics Compendium, 2012.

[12] EvaggelosSpyrou, Giorgos Stamou, Yannis Avrithis and Stefanos Kollias,” FUZZY SUPPORT VECTOR MACHINES FOR IMAGE CLASSIFICATION FUSING MPEG-7 VISUAL DESCRIPTORS”, Integration of Knowledge, Semantics and Digital Media Technology, 2005. EWIMT 2005. The 2nd European Workshop on the (Ref. No. 2005/11099).

[13] Ashish Ghosh, B. Uma Shankar, Saroj K. Meher "A novel approach to neuro-fuzzy classification”,Neural Networks, Volume 22, Issue 1, January 2009, Pages 100-10 9.

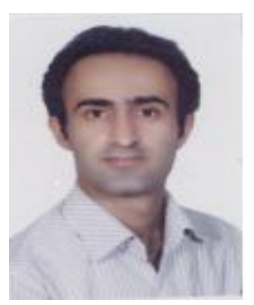

Mohammad Saber Iraji received B.Sc in Computer Software engineering from Shomal university, Iran, Amol;M.Sc1 in industrial engineering(system management and productivity) from Iran, Tehran and M.Sc2 in Computer Science. Currently, he is engaged in research and teaching on Computer Graphics, Image Processing, Fuzzy and Artificial Intelligent, Data Mining, Software engineering and he is Faculty Member of Department of Computer Engineering and Information Technology, Payame Noor University ,I.R. of Iran. 